\title{
Estimación del número reproductivo efectivo del brote de sarampión 2018-2019 en Chile
}

\author{
Estimation of the effective reproduction number of measles 2018-2019 in Chile
}

\author{
Mauricio Canals', Doris Gallegos ${ }^{2}$ y L. Fidel Avendaño 3
}

\begin{abstract}
${ }^{1}$ Programa de Salud Ambiental ESP y Departamento de Medicina. Facultad de Medicina. Universidad de Chile. ${ }^{2}$ Departamento de Epidemiología, Sub secretaría de Salud Pública._Ministerio de Salud. ${ }^{3}$ Programa de Virología. ICBM. Facultad de. Medicina. Universidad de Chile.
\end{abstract}

Financiamiento: No hubo.

Los autores declaran no tener conflicto de interés.

Recibido: 21 de agosto de 2019 / Aceptado: 4 de mayo de 2020

\section{Resumen}

Introducción: Los casos de sarampión están resurgiendo en muchos países del mundo. Hubo un brote de sarampión importado entre noviembre de 2018 y febrero de 2019 en Chile, lo que generó preocupación entre el público y las autoridades sanitarias. Muchos se preocuparon por la tasa de inmunización contra el sarampión de la población, un factor que se relaciona con la capacidad reproductiva del virus (medida de transmisibilidad de un patógeno). Objetivo: Aquí estimamos el número reproductivo efectivo $(R e)$ de este brote de sarampión. Resultados: Aunque la estimación tiene mucha incertidumbre por el bajo número de casos y la ausencia de mezcla homogénea de la población, encontramos que Re fue aproximadamente 1,5. Discusión y Conclusiones: En consecuencia estimamos que aproximadamente $90,3 \%$ de la población tiene inmunidad al sarampión, lo que coincide con las estimaciones del Ministerio de Salud. Estos resultados sugieren que la población chilena ha establecido la inmunidad colectiva contra la introducción de casos importados de sarampión, lo que refleja un manejo preventivo adecuado de esta enfermedad.

Palabras clave: Sarampión; número reproductivo; brote; inmunidad de rebaño.

\section{Introducción}

$\mathrm{E}$ n muchos países del mundo se continúa viviendo una importante alza en el número de casos de sarampión, incrementando en $300 \%$ en los tres primeros meses de 2019 , respecto al mismo período de 2018 ${ }^{1}$. La Organización Mundial de la Salud declaró en

\begin{abstract}
Background: Measles cases are reemerging in many countries across the globe. There was an outbreak of imported measles between November 2018 and February 2019 in Chile, raising concern among the public and health authorities. Many were worried about the Chilean measles herd immunity, a factor that relates to the reproductive capacity of the virus (measure of transmissibility of a pathogen). Aim: Here we estimate the effective reproductive number $(\mathrm{Re})$ of this measles outbreak. Results: Although the estimate is highly uncertain due to the low number of cases and the absence of homogeneous mixing of the population, we found Re was approximately 1.5. Discussion and Conclusions: Consequently we estimated about $90,3 \%$ had measles immunity, consistent with administrative estimates from the Ministry of Health. These results suggest the Chilean population has established herd immunity against the introduction of imported measles cases, reflecting adequate preventive management of this disease.

Keywords: Measles; effective reproduction number; outbreak; herd immunity.
\end{abstract}

2010 la necesidad de erradicar el sarampión y acelerar el control de la rubéola proponiendo obtener coberturas de vacunación de $80 \%$ o más con dos dosis y un sistema de vigilancia que asegure un alto nivel de inmunidad de rebaño en la población ${ }^{2}$. Chile ya había incorporado esas metas ${ }^{3}$ teniendo una vacunación programática con buenos niveles de cobertura y vigilancia sistemática de casos sospechosos basada 
en diagnóstico clínico y confirmación de laboratorio. Éstos le permitieron eliminar el sarampión autóctono en el país, a partir de 1993. Con posterioridad se han presentado solamente casos asociados a importaciones, los que no han desencadenado nuevos brotes asociados a importación. Sin embargo, siempre existe el riesgo de importación de casos asociado al fenómeno creciente de viajeros ${ }^{4-6}$. Entre noviembre de 2018 y febrero de 2019 ocurrió en Chile un brote de sarampión asociado a la importación que preocupó a la población y a las autoridades de salud ${ }^{7}$. Inmediatamente surgió la preocupación por el estado de inmunidad de la población para sarampión ${ }^{8}$, reforzando una serie de líneas de acción para controlar el brote.

En el análisis de brotes epidémicos, un concepto fundamental es el número reproductivo básico, que se define como el número de nuevos casos generados a partir de un caso cuando toda la población es susceptible ${ }^{9}$. El concepto de $R_{0}$ nació en la demografía. El concepto fue definido en su forma actual por Alfred Lotka $(1925)^{10}$ llamándolo "fertilidad neta". En epidemiología, la aproximación más robusta se encuentra en los trabajos de malaria de Ronald Ross quien identificó los principales factores en la transmisión de esta enfermedad y determinó el umbral de mosquitos bajo el cual la malaria no puede mantenerse en la población lo que es conocido como "mosquito theorem". Lotka, en un artículo llamado "una contribución a la epidemiología cuantitativa" reconoció la similitud de la reproducción de una población con la reproducción de una enfermedad estableciendo la conexión entre estos dos conceptos ${ }^{11}$. Posteriormente, Kermack y McEndrick, establecieron la conexión definitiva en 1927 entre el teorema del umbral (mosquito theorem) y $R_{0}{ }^{11}$. El número reproductivo no sólo tiene importancia epidemiológica; también se encuentra relacionado con la emergencia y evolución de enfermedades infecciosas ${ }^{12-14}$.

En el curso de una epidemia, la proporción de susceptibles va naturalmente decreciendo. Esto conduce a un decrecimiento de la probabilidad de nuevas infecciones. Es lo que Brownlee denominaba la dilución de los susceptibles en los casos y es la que explica por qué en una epidemia no se infectan todos los susceptibles más allá de las razones propias del estado inmunitario de cada individuo. Cuando no toda la población es susceptible ya sea por causas naturales o por medidas de control, el número reproductivo real no es exactamente $R_{0}$. Esto lo podemos expresar como $R_{e}=q R_{0}$, donde q representa la proporción de susceptibles, y $R_{e}$ es llamado el número reproductivo básico efectivo. Así, el $R_{e}$ es menor que $R_{0}$ cuando parte de la población está inmunizada y va decreciendo a medida que transcurre una epidemia, afectado por las medidas de control $^{9,15}$.

Si bien el $R_{0}$. depende prioritariamente de la naturaleza del agente, hay muchos otros factores que influyen, como la densidad de la población, la forma de contagio, la inmunidad previa, etc., por lo que actualmente se aconseja que cada país calcule el $R e^{5}$. A pesar de la existencia de una vacuna efectiva, las regiones del mundo han tenido un éxito variable en el control del sarampión, siendo una de las infecciones más contagiosas. Para el sarampión, $R_{0}$ a menudo se cita como $12-18$, lo que significa que cada persona con sarampión, en promedio, infectaría a 12-18 personas en una población totalmente susceptible ${ }^{5}$. En este trabajo estimamos el $R_{e}$ del brote de sarampión ocurrido en Chile entre noviembre de 2018 y febrero de 2019, y a partir de éste estimamos la proporción de población inmune.

\section{Material y Métodos}

Se analizó el total de casos del brote de sarampión entre noviembre de 2018 y febrero de 2019 obtenidos de la minuta de sarampión de febrero de 2019, emitida por el Ministerio de Salud ${ }^{7}$. Aunque existen varias aproximaciones para estimar el $R$ efectivo $\left(R_{e}\right)$ durante o después de epidemias ${ }^{9,16}$, el método más práctico es el estudio del aumento de los casos hasta el quiebre de la curva epidémica ${ }^{9,11,16}$. En este trabajo estimamos la tasa exponencial de aumento de los casos $(r)$ en una recta de regresión entre el logaritmo natural del número de casos y el tiempo: $\ln (\operatorname{Casos}+1)=r t+b_{0}$, donde $b_{0}$ es el intercepto y $r$ la pendiente de la recta de regresión. Se le agregó 1 al número de casos ya que no existe el logaritmo de cero; esto no afecta el valor de la pendiente. Como el número total de casos fue pequeño, puede haber variaciones al decidir cuál es el punto de quiebre, sin que el análisis pierda significación, por lo que se consideraron tres puntos de quiebre: $5^{\mathrm{a}}, 6^{\mathrm{a}}$ y $7^{\mathrm{a}}$ semanas: modelos T5, T6 y T7. Además, se consideró que en el brote existieron casos importados, por lo que se analizó también un modelo considerando exclusivamente los casos chilenos asociados a la importación desde el caso inicial, lo que es un modelo más exacto, aunque con escasos datos: modelo CH5. El parámetro $R_{e}$ se estimó mediante $R_{e}=1$ $+r \tau$, donde $\tau$ es el intervalo serial del sarampión, es decir, el tiempo promedio que transcurre entre una y otra infección. Este se ha estimado en promedio de 12 días = 1,71 semanas ${ }^{15,17}$. Se estimaron los intervalos de confianza para $R_{e}$ a partir de los intervalos de confianza de $r\left(\mathrm{IC}_{0.95}\right.$ $\left.r={ }_{{ }^{2} \mathrm{t}_{(1-\alpha / 2)}} e s(r)\right]$, donde es(r) es el error estándar de $r$. Considerando que $R_{e}=q R_{0}$, donde $q$ es la proporción de susceptibles en la población, se estimó la proporción de susceptibles mediante $q=R_{e} / R_{0}$, considerando que se ha reportado una mediana para el sarampión $R_{0}=15,6^{5} \mathrm{y}$ un rango habitual de variación entre 12 y $18^{18,19}$. De este modo, se pudo estimar la proporción de seroprevalencia o cobertura $p=1-q$ ( o 100(1-q) en porcentaje). 


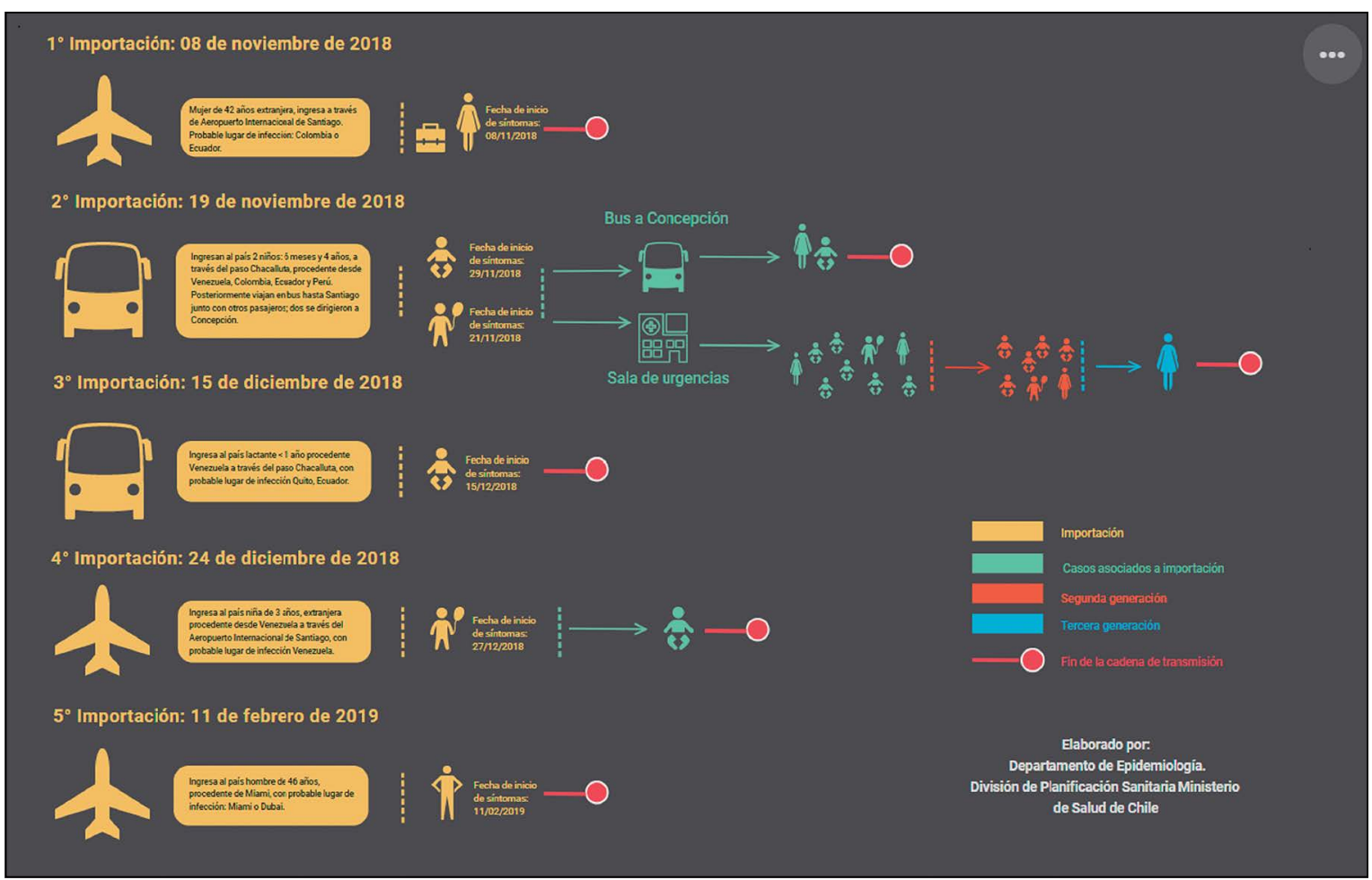

Figura 1. Cadena de transmisión de brote de sarampión asociado a importación. Noviembre 2018-febrero 2019. Fuente: Departamento de Epidemiología, DIPLAS, MINSAL.

\section{Resultados}

El brote de sarampión 2018/2019 fue de 26 casos y se extendió por 15 semanas (Figuras 1 y 2). Los parámetros estimados a partir de los modelos se presentan en la Tabla 1 . Hubo un buen ajuste de la regresión considerando el punto de quiebre a las 7 semanas (modelo T7) $\left(\mathrm{R}^{2}=0,67\right.$; $\mathrm{F}_{1,5}=10,3, \mathrm{p}=0,02$ ) (Figura 3). La tasa exponencial de crecimiento de los casos fue: $r=0,295 \pm 0,092$ (estimación \pm es) con un intervalo de confianza de $\mathrm{IC}_{0,95}=[0,059$; $0,532]$. Se consideró este valor como adecuado ya que los ajustes con puntos de quiebre a las 5 y 6 semanas proporcionaron valores similares de $\mathrm{r}: 0,346 \pm 0,191$ y $0,335 \pm$ 0,126 para 5 y 6 semanas, respectivamente. Por otra parte, los valores de los parámetros estimados con este modelo son prácticamente iguales a los estimados considerando sólo los casos de transmisión local (modelo CH5).

A partir del modelo T7, la estimación de $R_{e}$ fue: $R_{e}=1,506$ con un $\mathrm{IC}_{0,95}=[1,10 ; 1,91]$, lo que implica una proporción de susceptibles $\mathrm{q}=100(1,506 / 15,6)=9,65 \%$ y un porcentaje de cobertura $\mathrm{p}=90,34 \%$ con un intervalo de confianza $\mathrm{IC}_{0,95}=[87,8 ; 92,9] \%$.

La sensibilidad de las estimaciones al valor de $R_{0}$ usando los valores extremos del rango para todos los modelos muestra que $\mathrm{p}$ puede variar entre un mínimo de $78 \%$ y un máximo de $94,4 \%$.

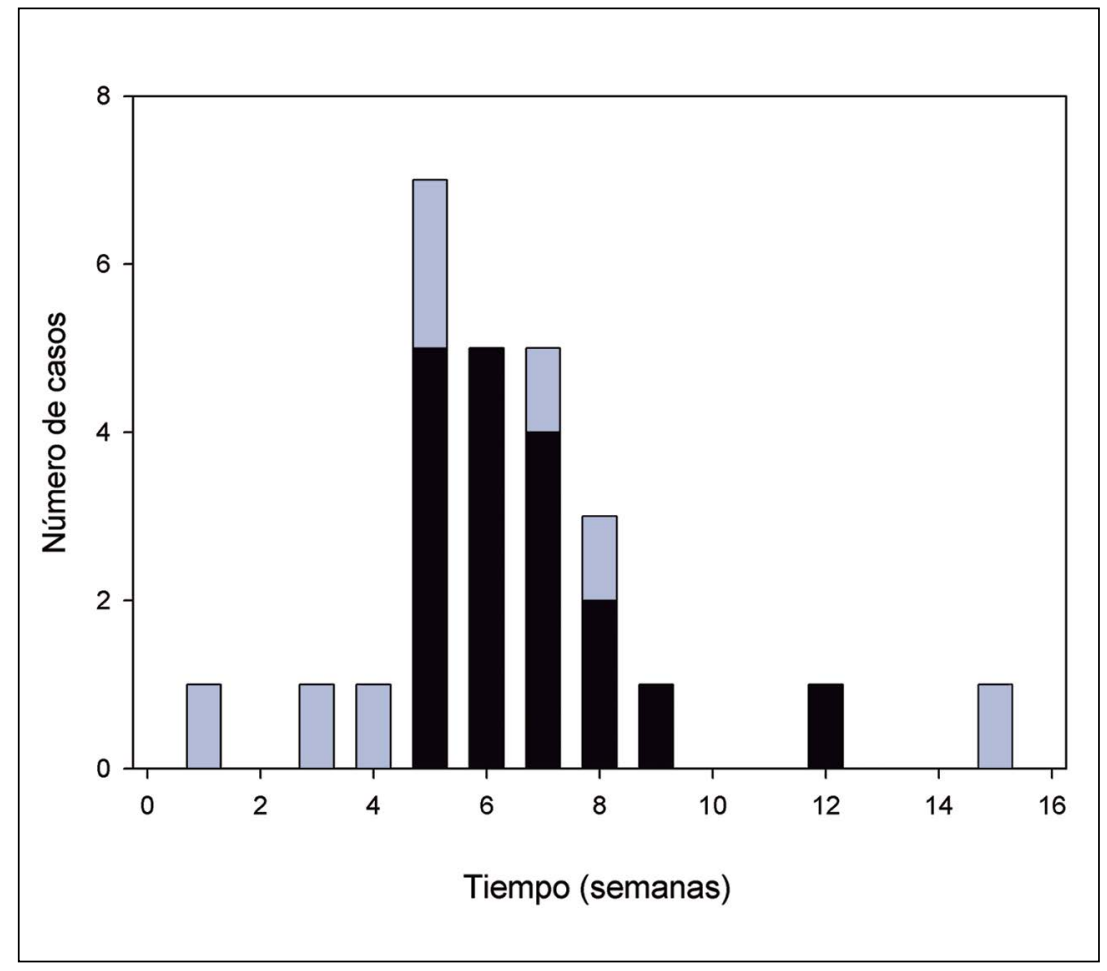

Figura 2. Brote de sarampión en Chile 2018-2019. Casos nuevos por semana. En gris los casos importados, en color negro los casos autóctonos asociados a la introducción. Fuente MINSAL?. 


\section{— W I Epidemiología}

\begin{tabular}{|c|c|c|c|c|c|c|c|c|}
\hline Modelo & $r$ (estima \pm es) & $\mathbf{R}^{2}$ & $F(g . l)$. & p & $\boldsymbol{R}_{e}$ & $\mathrm{IC}_{0,95} \boldsymbol{R}_{\mathrm{e}}$ & $p$ & $\mathrm{IC}_{0,95} \mathrm{P}$ \\
\hline T5 & $0,347 \pm 0,192$ & 0,52 & $3,26(1,3)$ & 0,169 & 1,59 & $1-2,64$ & 89,8 & $83,1-93,6$ \\
\hline T6 & $0,335 \pm 0,126$ & 0,64 & $7,1(1,4)$ & 0,056 & 1,57 & $1-2,17$ & 89,9 & $86,0-93,6$ \\
\hline T7 & $0,295 \pm 0,092$ & 0,67 & $10,32(1,5)$ & 0,023 & 1,51 & $1,1-1,91$ & 90,3 & $87,8-92,9$ \\
\hline $\mathrm{CH} 5$ & $0,293 \pm 0,123$ & 0,65 & $5,66(1,3)$ & 0,098 & 1,50 & $1-2,17$ & 90,4 & $86,1-93,6$ \\
\hline
\end{tabular}

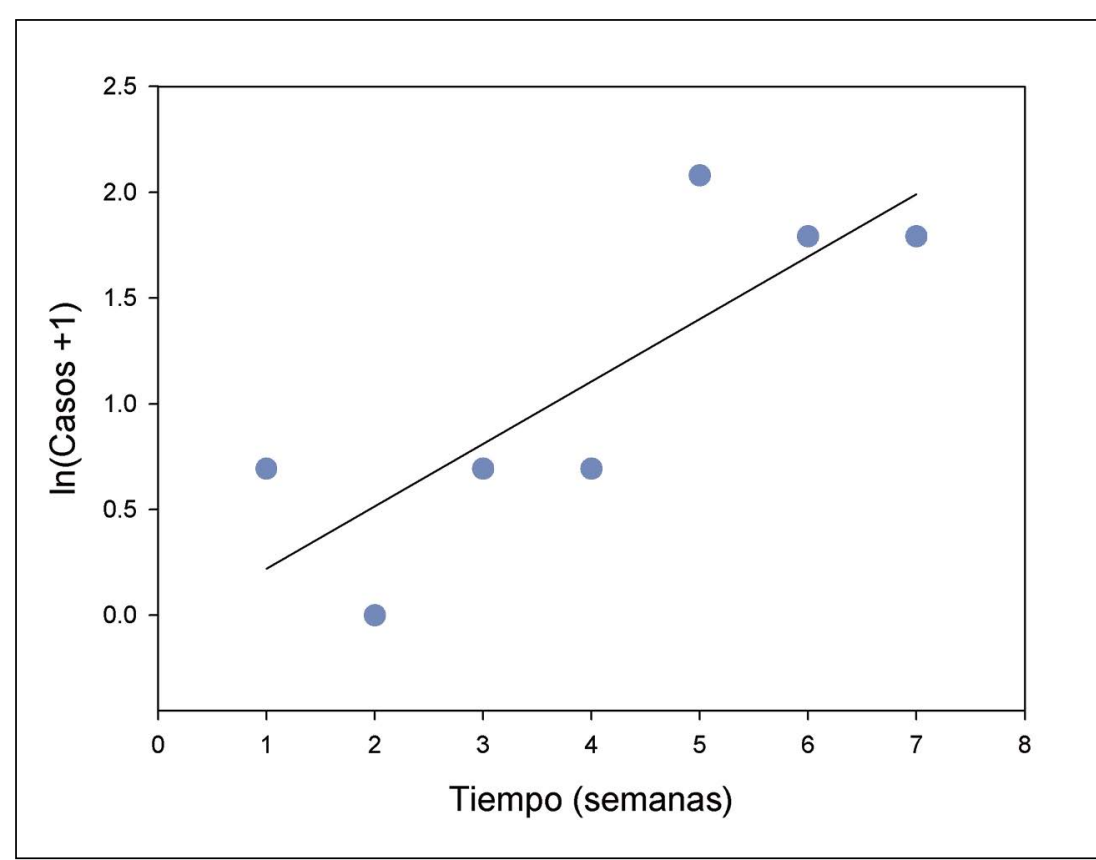

Figura 3. Ajuste de la recta de regresión (línea continua) al logaritmo natural de los casos $(\ln ($ Casos +1$))$ versus tiempo en las primeras siete semanas (modelo T7).

\section{Discusión}

La estimación de $R_{e}$ del brote 2018/2019 fue bastante consistente en todos los modelos indicando un $R$ de alrededor de 1,5 lo que señala una seroprevalencia de la población de alrededor de 90,3\%. Este valor es consistente con los cálculos del Ministerio de Salud de 89,5\% basados en la población entre 15 y 40 años a nivel nacional, según la Encuesta Nacional de Salud 2015-2016 $6^{8}$. Sin embargo, los valores estimados son sensibles al valor del $R_{0}$ usado en los cálculos, obteniéndose valores de al menos $78 \%$ para $R_{0}=12$ y de al menos $94,4 \%$ para $R_{0}=18$, considerados el rango habitual para el sarampión.

Otras aproximaciones para el cálculo de $R_{0}$ son $R_{0}=\ln (1 / \mathrm{s}) /(1-\mathrm{s})$ que supone conocer el valor de " $s$ " que representa el número de susceptibles al final de la epidemia, el que sólo puede ser estimado al final del brote y sólo en poblaciones cerradas, lo que lo hace poco útil. Otra aproximación se puede hacer con la relación $R_{0}=1$ $+\left(e_{d} E\right)$, donde $e_{0}$ es la esperanza de vida al nacer de la población y $E$ es la edad media de infección que supone un equilibrio endémico y una mezcla homogénea de la población, lo que hace que en pequeños brotes no sea útil $1^{9}$.

El método usado en este trabajo, tiene algunas limitaciones. Por una parte, supone una mezcla homogénea de la población, requisito que en general no se cumple pues los brotes pequeños no se extienden por todo Chile $\mathrm{y}$, por otra parte, supone un crecimiento exponencial de los casos al inicio del brote'. Este último supuesto se cumple si hay un adecuado ajuste de la regresión, lo que en nuestro caso ocurrió en el modelo T7. Otro problema se encuentra en la decisión de cuál es el punto de quiebre de la curva epidémica. Observando la Figura 1, parece existir un quiebre natural de la curva a las 5 semanas (modelo T5); sin embargo, en la semana 6 y en la 7 hubo 5 casos y se dificulta decidir si esta diferencia es real o es una variación aleatoria. En la semana 8 efectivamente existió una clara reducción de casos. Otro problema es el número de puntos (semanas consideradas) para ajustar el modelo. Mientras menor sea éste, menores son los grados de libertad y entonces se pierde la significación estadística. En teoría, el modelo más correcto es aquel que considera sólo los casos chilenos asociados al brote y no las importaciones (modelo CH5); sin embargo, al considerar este modelo hay que incluir el caso que da inicio al brote que es importado y además se obtiene con pocos puntos. A pesar de todos estos problemas, sin considerar la significación estadística, todos los modelos fueron muy consistentes en todos los parámetros, lo que aporta bastante seguridad en las estimaciones reportadas.

El brote ocurrió desde mediados de noviembre de 2018 a febrero de 2019 , confirmándose 26 casos, entre los cuales se detectaron cinco importaciones distintas al país, con la mayoría de los casos relacionados a la segunda importación en la Región Metropolitana. El 50,7\% de los casos fue en lactantes menores de 1 año, $16 \%$ entre 1 y 14 años y $27 \%$ en mayores de 14 años. Este brote fue declarado interrumpido a mediados de abril. 
es decir, aproximadamente entre 3,5 y $3,7 \%$.

Es importante comentar que este tipo de estimaciones se basa en una mezcla homogénea de la población y no considera la heterogeneidad espacial de ésta. En este caso, el pequeño brote se debe fundamentalmente a la segunda importación, que participó en la elevación de casos, con poca participación de las importaciones posteriores. Un valor de $R_{e}=1,51$ (con $\mathrm{IC}_{0,95}[1,1-1,91]$ ) puede parecer un poco alto; sin embargo, hay que considerar que el concepto de $R_{0}$ se refiere a la capacidad de dispersión del agente infeccioso cuando se enfrenta a una población homogénea y totalmente susceptible, de tal manera que hace altamente probable que la infección se instale en la población y genere una epidemia. Por el contrario, en una población no homogénea y con una alta cobertura de vacunas, donde los susceptibles se encuentran disgregados y dispersos, pueden existir pequeños brotes con esta capacidad de contagio, pero que se resuelven rápidamente, lo que parece ser el caso de este pequeño brote de sarampión.

El $R_{e}$ es una medida de la transmisión de una enfermedad en la población y es, a la vez, una medida de lo adecuado de las medidas de control y mitigación de un brote. El $R_{e}$ decae en el curso de una epidemia. Un ejemplo de este efecto se pudo observar en la pandemia A(H1N1)-2009, lo que se atribuyó a la implementación de las medidas de control antes que a la dilución de los susceptibles, ya que el número de casos en esta etapa es muy inferior a la gran abundancia de susceptibles ${ }^{22}$.

En el caso del sarampión, el $R_{e}$ tan bajo comparado al $R_{0}$ natural de la afección es una indicación del buen manejo del control de esta enfermedad en Chile, que sólo tiene casos esporádicos desde 1993 a partir de la predicción de epidemias y las eficientes campañas de vacunación a partir de los $90^{\prime} \mathrm{s}^{6,23}$, lo que además conduce a una baja probabilidad de contagio de viajeros chilenos en el extranjero ${ }^{6}$.

\section{Referencias bibliográficas}

1.- WHO 2019. New measles surveillance data for 2019. https://www.who.int/immunization/ newsroom/measles-data-2019/en/ (consultada en julio de 2019).

2.- WHO. Global Measles and Rubella Strategic Plan: 2012-2020. World Health Organization 2012. ISBN 978924150339 6. https://apps.who.int/iris/bitstream/ handle/10665/44855/9789241503396_eng.pdf; jsessionid=3FE1A95F43340325D638D256434 BC9D0?sequence $=1$.

3.- Gallegos D. Ministerio de Salud Chile Instituto de Salud Pública-Organización Panamericana de la Salud. Documentación para la verificación de la eliminación del sarampión, rubéola y síndrome de rubéola congénita en Chile. 2011. http://www.repositoriodigital. minsal.cl/bitstream/handle/2015/461/ sarampion.pdf? sequence $=1$ \&isAllowed $=y$.

4.- Da Silva L H, Minoru Fujita D, Scassi Salvador F, Domínguez Da Silva Fink M C, Franco de Andrade H, Sérgio Pannuti C, et al. Potential measles transmission risk in mass gathering: are we safe for the Olympic games- Rio 2016 J Travel Med 2016; 23: 1-3. doi: 10.1093/jtm/ taw026.

5.- Guerra F M, Bolotin S, Lim G, Heffernan J, Deeks S L, Li Y, et al. The basic reproduction number (Ro) of measles: a systematic review. Lancet Infect Dis 2017; 17: e420 -428. doi: 10.1016/S1473-3099(17)30307-9.

6.- Canals M, Rojas C, Avendaño L F. Riesgo de contagio de sarampión para un viajero chileno en 2017. Rev Med Chile. 2019; 147: 650-7. http://dx.doi.org/10.4067/S003498872019000500650 .

7.- MINSAL. Brote de sarampión asociado a importación. Chile, noviembre 2018 - febrero 2019 www.minsal.cl (14/2/2019). http:// epi.minsal.cl/wp-content/uploads/2019/02/ MINUTA_SARAMPI\%C3\%93N_2019_02_14 pdf.

8.- MINSAL. Detección de nuevo caso importado de sarampión en chilena que se contagia en Europa. Junio 2019 www.minsal.cl $(19 / 6 / 2019)$

9.- Canals M. Introducción a la epidemiología matemática. Santiago, Chile: Edición Sociedad Chilena de Parasitología; 2017. 
10.- Lotka A. Elements of physical biology. Baltimore, United States: Williams \& Wilkins; 1925.

11.- Heffernan J M, Smith R J, Wahl L M. Perspectives on the basic reproductive ratio. J. R. Soc. Interface; 2015: 1-13. doi: 10.1098/ rsif.2005.0042.

12.- Lloyd-Smith J, Dylan G, Pepin K M, Pitzer V E, Pullian J R, Dobson A P, et al. Epidemic dynamics at the human-animal interface. Science 2009; 326: 1362-7. doi: 10.1126/ science. 1177345 .

13.- Wolfe N D, Dunaban C P, Diamond J. Origins of mayor human infectious diseases. Nature 2007; 447: 279-83. doi: 10.1038/nature05775.

14.- Canals M, Torres-Contreras H, Castro SA, Jaksic F M. 2013. Dinámica poblacional de la expansión de enfermedades infecciosas como modelo de invasiones biológicas. En: Invasiones Biológicas. (Castro SA \& Jaksic FM Eds). Ediciones P. Universidad Católica de Chile. Pp 459-91.
15.- Paterson B J, Kirk M D, Scott Cameron A, D’Este C, Durrheim D N. Historical data and modern methods reveal insights in measles epidemiology: a retrospective closed cohort study. BMJ Open. 2013; 3: e002033. doi:10.1136/bmjopen-2012-002033.

16.- Canals M, González C R, Canals A, Figueroa D P. Dinámica epidemiológica del dengue en Isla de Pascua. Rev Chilena Infectol 2012; 29 (4): 388-94. http://dx.doi.org/10.4067/S071610182012000400004.

17.- Vink M A, Bootsma M C J, Wallinga J. Serial intervals of respiratory infectious diseases: a systematic review and analysis. Am J. Epidemiol. 2014; 180(9): 865-75. doi: 10.1093/ aje/kwu209.

18.- Anderson R M, May R. Vaccination against rubella and measles: quantitative investigations of different policies. J Hyg Camb 1983; 90 : 259-325. doi: 10.1017/s002217240002893x.

19.- Fine P E M, Mulholland K, Scott J A, Edmunds W J. Community protection. En:
Plotkin's Vaccines $7^{\text {th }}$ edition. Plotkin SA, Orenstein WA, Offit PA., Edwards KM, eds. Philadelphia: Saunders Elsevier; 2018. p. 1512-31.

20.- Chowell G, Fuentes R, Olea A, Aguilera X, Nesse H, Hyman J N. The basic reproduction number R0 and effectiveness of reactive interventions during dengue epidemics: The 2002 dengue outbreak in Eastern island, Chile. Math Biosc. Eng. 2013; 10: 1455-74. doi: $10.3934 / \mathrm{mbe} .2013 .10 .1455$.

21.- CDC. Transmission of measles. https://www. cdc.gov/measles/transmission.html (consultado el 20 de agosto de 2019).

22.- Canals M. Análisis comparado de la dinámica epidemiológica de la influenza A(H1N1) en Chile. Rev Med Chile 2010; 138:117989. http://dx.doi.org/10.4067/S003498872010000900016.

23.- Canals M. Sarampión en Chile: Una peligrosa onda. Rev Med Chile 1992; 120: 585-8. PMID: 1343073. 